\title{
WOMEN'S PERCEPTION OF NURSE'S ROLE IN CESAREAN SECTION CARE
}

\author{
*Nora Abd Allah Howiady, **Prof. Mohamed Mokhtar Shaaban, ***Dr. Inas \\ Mohamed Abd Allah, ****Dr.Mervat Elshahat Ebrahim Shelil \\ *B.Sc. Nursing,, Faculty of Nursing, Suez Canal University, noor3eyny@ yahoo.com, \\ **Professor of Obstetrics \&Gynecology Medicine, Faculty of Medicine, Suez Canal \\ University, Assistant Professor, Maternity, Obstetric \& Gynecological Nursing, Faculty \\ of Nursing, Suez Canal University, Lecturer of Family\& Community Health Nursing, \\ Faculty of Nursing, Suez Canal University
}

\begin{abstract}
Background: Women as customer in obstetric field are considered important source of information for improving nursing care provided. Women's perceptions of nursing care, especially in the obstetric field, are not well known. Aim of the study was to identify women's perception of nurse's role in cesarean section care. It was carried out at the obstetric and gynecological department of Suez Canal University hospital and Ismailia general hospitals in Ismailia city. Research questions: How are women's perceived pre and post-operative nurse's role in C.S care? And what are the factors affecting women's perception? Design: a descriptive correlational design was adopted. Sample: purposive, non-probability sampling was followed to recruit 200 full term uncomplicated pregnant women who undergone cesarean section. Tool of data collection: semi-structured interviewing questionnaire was used. It covers women's perception about nurse's role and factors may affect their perception. Results: Women's perceptions of pre-operative nurse's role (as physical care, emotional support \&communication and providing of necessary information) were accepted by the highest frequency of women while the postoperative nurse's role (as; availability of bedside nurse, emotional support \& communication, providing necessary information and providing baby care) was described as poor care. Urban residence, multiparty, antenatal care, and setting of delivery are significant predictors of good woman's perception $(\mathrm{P}<0.001)$. Conclusion; Women's perceptions of pre-operative nurse's role were accepted care, while postoperative nurse's role was described as poor care. Recommendation: Nursing staff should take into consideration the patients' perception so as to meet patients' needs
\end{abstract}

key words : Cesarean Section; Women's Perception, Nurse's Role 


\section{إدراك السيدات لاور الممرضة في العناية بالعملية القيصرية

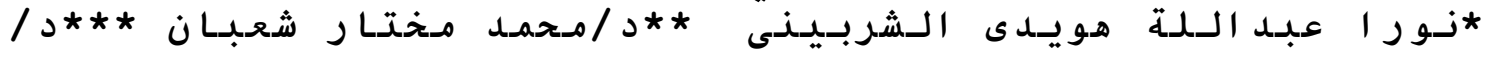

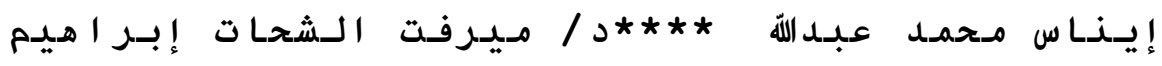

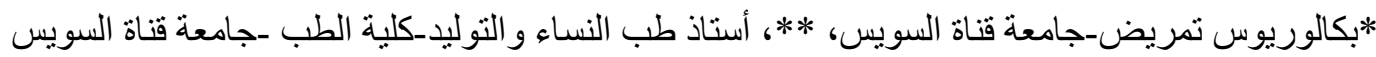

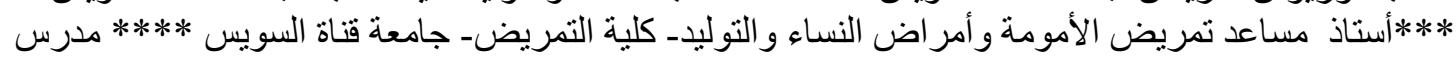

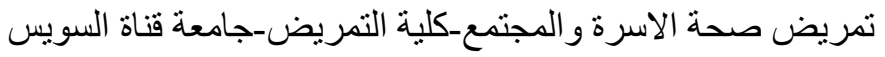 \\ الملخص}

إدراك السيدات عن الرعاية التمريضية ماز ال غير معروف فى مجال النساء والتوليد ولذلك فالسيدات هم أهم مصادر للمعلومات لتحسين الرعاية التمريضية فى هذا المجال. الهوف من اللدراسة: : تهدف هذة الدراسة إلى التعرف على إدراك السيدات لدور الممرضة فى العناية بالعملية القيصرية. عينة ومكان البحث: تضمنت عينة هذا البحث 200 من الأمهات اللاتي وضعن اطفال عن طريق العمليات القيصريه في مستشفى جامعة قناة السويس و المستثفي العام بالاسماعيلية وكان اختيار السيدات من الائى حضرن إلى المستثفى بغرض إجراء العملية القيصرية و تم استبعاد السيدات اللاتي لديهم مضاعفات قبل او بعد العملية والائى حدث وفاة المولود قبل أو أثناء أو بعد الو لاده مباشرة. أدوات البحث : تضمنت أدوات البحث على : استمارة إستبيان عن أدرالك السيات لدور الممرضة والعوامل التي تؤثر علي ادر اكهن. وقد أسفرت نتائج البحث على: كان إدراك السيدات للاور التمريضى قبل العملية يعتبر مقبو لا بينما كان إدراك السيدات للدور التمريضى بعد العملية القيصرية ردئ . يعتبر كبر السن، زيادة عدد مرات الحمل وزيادة عدد مرات متابعة الحمل بالمستشفى الجامعى من العوامل ذات الدلالة الاحصائية التي حسنت ادر اك السبدات للدور التمريضى المقدم. ولثذا بيوصي بالأتي يجب الاخذ فى الاعتبار إدراك السيدات للر عاية التمريضية التى تتم من خلال الفريق التمريضى لمقابلة إحتياجاتهن. الكلمات الإسترشادية:العملية القيصرية; إدر الك السيدات ;الدور التمريضى 